\title{
Commentary on the Paper by Wang et al: "Low Arousal Threshold: A Potential Bridge Between OSA and Periodic Limb Movements of Sleep" [Letter]
}

This article was published in the following Dove Press journal:

Nature and Science of Sleep

\section{Mauro Manconi (DiD ${ }^{1-3}$ Raffaele Ferri $\mathbb{D}^{4}$}

'Sleep Medicine Unit, Neurocenter of Southern Switzerland, Lugano, Italy; ${ }^{2}$ Faculty of Biomedical Sciences, Università della Svizzera Italiana, Lugano, Switzerland; ${ }^{3}$ Department of Neurology, University Hospital, Inselspital, Bern, Switzerland; ${ }^{4}$ Sleep Research Centre, Oasi Research Institute - IRCCS, Troina, Italy
Correspondence: Mauro Manconi Email mauro.manconi@eoc.ch

\section{Dear editor}

We have read with great interest the paper by Wang et al ${ }^{1}$ that shed a welcome spot of light on the intriguing and poorly addressed linkage between periodic limb movements (PLMS) and obstructive sleep apnea (OSA). With an elegant, despite retrospective, investigation, they tested the hypothesis that the OSA phenotype associated with low arousal threshold (ArTH) was a risk factor for the cooccurrence of OSA and PLMS. Once having rejected the null hypothesis, the authors confirmed their results in the independent large population of the MrOS study, and this is certainly a strength of the study. A second noteworthy methodological merit of the authors is to have used the new data-driven criteria to define the so-called respiratory-related leg movements (RRLM) proposed by the WASM taskforce. ${ }^{2}$ By using these criteria, all leg movements (LM) occurring within the time range from $2 \mathrm{~s}$ before to $10.25 \mathrm{~s}$ after the end of respiratory events were defined as RRLM. In addition, the authors repeated their analysis also using the standard AASM criteria (RRLM = LM overlapping or not separated by more than $\pm 0.5 \mathrm{~s}$ from the end of the respiratory event). ${ }^{3}$ Since the recording of LM has become a standard procedure in accredited sleep labs, PLMS and RRLM are a frequent incidental finding and, besides their clinical significance possibly suggesting the existence of an underlying restless legs syndrome, the value of such motor components remains unclear as well as the option to treat them with dopamine agonists (DA).

Some methodological and interpretative aspects of the study warrant a more indepth discussion.

First, the surrogate criteria to establish the "low-threshold" OSA phenotype is based fundamentally on criteria of low severity, being identified by a low AHI, a non-deep desaturation and on the high proportion of hypopnea vs apnea events. ${ }^{4}$ At the same time, PLMS in OSA are also intrinsically linked with a low respiratory severity pattern, this regardless of the ArTH. In fact, in a patient affected by OSA, for definition, PLMS series may occur only outside of the sequences of breathing events. Therefore, in a patient with highly severe OSA, in whom most of the sleep time is occupied by pathological breathing events, PLMS might simply not 
manifest themselves since there is no available "space" and, if they occur, they would be excluded by definition, because they are classified as RRLM. This is an intrinsic bias that tends to segregate PLMS with low severity OSA, especially if the threshold of $15 /$ hour is used instead of the old threshold of 5/hour for the classification of subjects as affected by PLMS. However, the authors correctly stressed the fact that the definition of low ArTH in their population was not based on the criteria of $\mathrm{AHI}<30$, but on the other two criteria (fraction of hypopneas and minimum desaturation). The latter are, however, also indicative of a low OSA severity, as confirmed in their table 1 in which the oxygen desaturation index (ODI) was significantly lower in the OSA-PLMS group.

Second, an additional aspect of the study concerns the analysis of the respiratory pattern, which was restricted to apnea and hypopnea events, without considering respiratory effort-related arousals (RERA) and without discriminating between obstructive and central hypopneas, and between breathing events occurring in NREM or REM sleep. The key element of RERA is represented by the "arousal" that can be triggered or at least enhanced in its duration and amplitude by an underlying associated LM. Vice versa, the arousal itself might favor a leg motor response. Central hypopnea often accompany the lowarousal-threshold OSA phenotype, being promoted by unstable sleep and the associated phase of hyperventilation, without forgetting that also central breathing events might end with an LM. ${ }^{5}$ Finally, the so-called "REM-OSA phenotype" (patients with most of the events segregated in REM sleep) need to be quantified, since, as already well known, PLMS have a clear relationship with sleep stage, tending to decrease in REM sleep and to increase in NREM sleep S2.

Third, the concept of separating PLMS from RRLM, relies on their possible and supposed different pathogenesis. RRLM are thought to be triggered by the respiratory event per se, while PLMS by a different still unclear individual predisposition or by a hypothetical central dopaminergic dysfunction. This dichotomy, based on a conjectural diversity of the nature of these two types of events, is still not supported by solid evidence. In a seminal paper on RRLM in moderate OSA, ${ }^{6}$ we showed that almost only patients able to produce regular non respiratory related PLMS, could additionally produce RRLM. In other words, patients without PLMS, usually do not present RRLM. We found that, in OSA patients with RRLM, the intermovement interval distribution of the total LM activity during sleep shows three distinctive peaks: the typical first peak within $5 \mathrm{~s}$ (short interval LM), the typical second peak around 15-25 s referred to PLMS, and an additional third more platykurtic peak occurring at around 30-40 s, reflecting the RRLM. Patients with OSA and without the second peak (PLMS) usually also lack the third one (RRLM) (Figure 1). This result suggests that RRLM might simply be normal PLMS that are captured by the respiratory events and forced to occur within their rhythm; thus, respiratory events might act as time synchronizers and not as a causal determinants of RRLM. This also explain why the effect of CPAP is highly heterogeneous and, often, patients continue to have PLMS, sometimes with an increased PLMS index. ${ }^{7}$ Herein, it would be extremely interesting to explore the genetic background of patients with RRLM, in order to verify the prevalence among them of the allelic variants already known to be associated with PLMS, such as BTBD9 and MEIS1. ${ }^{8}$

Finally, we thank the authors for having considered our pharmacological study in RLS patients, with which we challenged the concept of a cause/effect relationship between PLMS and cortical arousals. ${ }^{9}$ We showed that the suppression of PLMS by using dopamine-agonists was not accompanied by a similar reduction in arousals, as it could be expected. However, the authors did not mention the second part of the study demonstrating that a suppression of cortical arousability, by clonazepam, was not associated to a reduction in PLMS. If, on one side, the persistence of arousals might represent a risk factor for cardiovascular disease, on the other hand, it could be interpreted as a phenomenon not supporting the hypothesis that a low arousal threshold is associated with the presence of PLMS. Indeed, our study was conducted in subjects with RLS and without OSA.

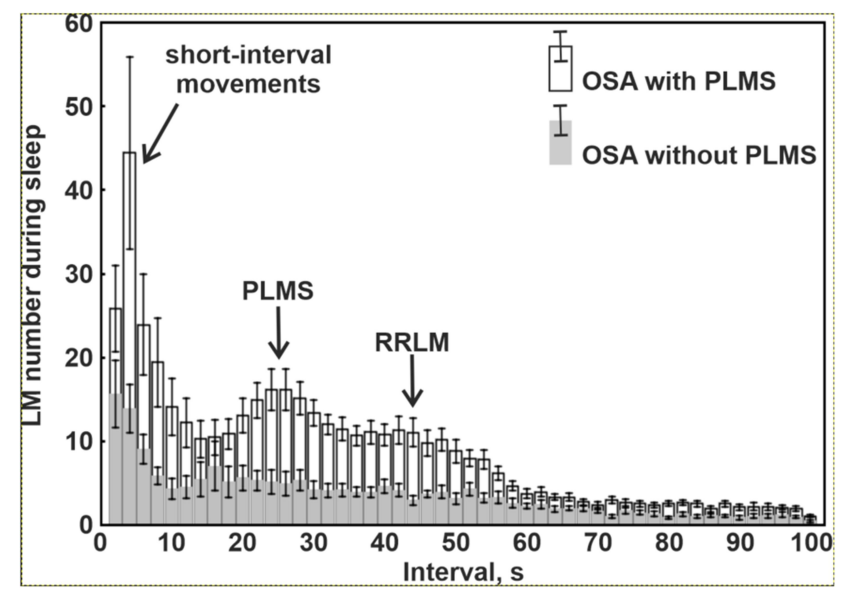

Figure I Comparison of the distribution of LM intermovement intervals during sleep in patients with OSA and with or without PLMS. This figure has been redrawn based on data reported by Manconi et al in $2014 .^{6}$ 
In conclusion, we really appreciate the paper by Wang et $\mathrm{al}^{1}$ and we encourage the authors and other researchers to keep exploring this topic, but also suggest to provide more details on RRLM, RERA, REM-related respiratory events, and even better to depict the inter-movement distribution of the whole LM activity during sleep. A confirmation of these results, would candidate PLMS as a possible marker of the "low ArTH" OSA phenotype, while the link between RRLM and PLMS in OSA and cardiovascular disease requires much more research with longitudinal and interventional trials.

\section{Disclosure}

The authors report no conflicts of interest in this communication.

\section{References}

1. Wang Q, Li Y, Li J, et al. Low arousal threshold: a potential bridge between OSA and periodic limb movements of sleep. Nat Sci Sleep. 2021;13:229-238. doi:10.2147/NSS.S292617
2. Ferri R, Fulda S, Allen RP, et al. World Association of Sleep Medicine (WASM) 2016 standards for recording and scoring leg movements in polysomnograms developed by a joint task force from the International and the European Restless Legs Syndrome Study Groups (IRLSSG and EURLSSG). Sleep Med. 2016;26:86-95. doi:10.1016/j.sleep.2016.10.010

3. Iber C. The AASM manual for the scoring of sleep and associated events: rules. Terminol Tech Spec. 2007.

4. Aishah A, Eckert DJ. Phenotypic approach to pharmacotherapy in the management of obstructive sleep apnoea. Curr Opin Pulm Med. 2019;25:594-601. doi:10.1097/MCP.0000000000000628

5. Manconi M, Vitale G, Ferri R, Zucconi M, Ferini-Strambi L. Periodic leg movements in Cheyne-Stokes respiration. Eur Respir J. 2008;32:1656-1662. doi:10.1183/09031936.00163507

6. Manconi M, Zavalko I, Bassetti CL, et al. Respiratory-related leg movements and their relationship with periodic leg movements during sleep. Sleep. 2014;37:497-504. doi:10.5665/sleep.3484

7. Budhiraja R, Javaheri S, Pavlova MK, et al. Prevalence and correlates of periodic limb movements in OSA and the effect of CPAP therapy. Neurology. 2020;94:e1820-e1827. doi:10.1212/WNL.00000000000 08844

8. Jiménez-Jiménez FJ, Alonso-Navarro $\mathrm{H}$, García-Martín $\mathrm{E}$, Agúndez JAG. Genetics of restless legs syndrome: an update. Sleep Med Rev. 2018;39:108-121. doi:10.1016/j.smrv.2017.08.002

9. Manconi M, Ferri R, Zucconi M, et al. Dissociation of periodic leg movements from arousals in restless legs syndrome. Ann Neurol. 2012;71:834-844. doi:10.1002/ana.23565

Dove Medical Press encourages responsible, free and frank academic debate. The content of the Nature and Science of Sleep 'letters to the editor' section does not necessarily represent the views of Dove Medical Press, its officers, agents, employees, related entities or the Nature and Science of Sleep editors. While all reasonable steps have been taken to confirm the content of each letter, Dove Medical Press accepts no liability in respect of the content of any letter, nor is it responsible for the content and accuracy of any letter to the editor.

\section{Publish your work in this journal}

Nature and Science of Sleep is an international, peer-reviewed, open access journal covering all aspects of sleep science and sleep medicine, including the neurophysiology and functions of sleep, the genetics of sleep, sleep and society, biological rhythms, dreaming, sleep disorders and therapy, and strategies to optimize healthy sleep.
The manuscript management system is completely online and includes a very quick and fair peer-review system, which is all easy to use. Visit http://www.dovepress.com/testimonials.php to read real quotes from published authors. 